19 Revue d'histoire du XIXe siècle

Société d'histoire de la révolution de 1848 et des

révolutions du XIXe siècle

$57 \mid 2018$

Libido sciendi

Priya SATIA, Empire of Guns. The Violent Making of the Industrial Revolution

François Jarrige

URL : https://journals.openedition.org/rh19/6227

DOl : $10.4000 /$ rh 19.6227

ISSN : $1777-5329$

Éditeur

La Société de 1848

Édition imprimée

Date de publication : 26 décembre 2018

Pagination : 214-217

ISSN : 1265-1354

Référence électronique

François Jarrige, "Priya satıA, Empire of Guns. The Violent Making of the Industrial Revolution », Revue d'histoire du XIXe siècle [En ligne], 57 | 2018, mis en ligne le 26 décembre 2018, consulté le 21 janvier 2022. URL : http://journals.openedition.org/rh19/6227 ; DOI : https://doi.org/10.4000/rh19.6227

Ce document a été généré automatiquement le 21 janvier 2022.

Tous droits réservés 


\title{
Priya SATIA, Empire of Guns. The Violent Making of the Industrial Revolution
}

\author{
François Jarrige
}

\section{RÉFÉRENCE}

Priya Satia, Empire of Guns. The Violent Making of the Industrial Revolution, Londres, Penguin, 2018, 544 p., \$ 35.

1 Professeure d'histoire à l'Université de Stanford aux États-Unis, Priva Satia est reconnue pour ses travaux sur l'action de la Grande-Bretagne au Moyen-Orient autour de la Première Guerre mondiale ${ }^{1}$. "L'empire des armes" est son second livre, il poursuit et élargit ses recherches antérieures sur l'histoire des technologies et de la violence dans l'empire britannique. L'ouvrage est particulièrement important et ambitieux puisqu'il couvre trois siècles et explore les relations qui n'ont cessé de se nouer entre les guerres, l'impérialisme et les mutations économiques de l'Occident. Il offre une relecture originale des origines et des formes de l'industrialisation britannique en les inscrivant dans une perspective globale et impériale désormais bien installée, et surtout en les reliant à la culture de la violence et à l'histoire des armes à feu et de leur fabrication.

2 L'étude démarre avec l'évocation du dilemme moral de Samuel Galton, un quaker également à la tête de l'une des principales manufactures d'armes britanniques, qui fut aussi membre de la Royal Society et de la Lunar Society. À la fin du xviII siècle, les quakers dénoncent cette activité jugée immorale et contraire aux principes chrétiens. Galton se justifie en invoquant l'utilité des armes pour la défense de la civilisation, mais aussi leur rôle central dans la prospérité du pays. En étudiant de près les armes, leurs fabrications, leur circulation et leurs utilisations aux XVIII ${ }^{\mathrm{e}}$ et XIX ${ }^{\mathrm{e}}$ siècles, Priva Satia propose de relire les origines du capitalisme industriel à l'aune des armes plutôt qu'à 
celle du doux commerce et de l'essor de l'industrie textile auxquels on ramène en général le processus. En faisant des armes à feu un observatoire privilégié pour examiner l'évolution des imaginaires et des pratiques économiques en GrandeBretagne, l'auteure offre une analyse riche et rafraichissante de transformations industrielles qu'on pensait bien connaître. La dernière phrase de l'introduction résume bien l'enjeu du livre: «Nous avons oublié le poids de l'industrie des armes dans l'essor du capitalisme industriel, comme nous oublions le sang qui coule dans nos veines». C'est cet oubli qu'il s'agit de réparer pour éclairer les fondations de notre monde.

3 Si les liens entre les guerres mondiales $\mathrm{du} \mathrm{xx}^{\mathrm{e}}$ siècle et l'avènement des technosciences contemporaines ont été étudiés par l'histoire des sciences et de l'environnement, ceux qui ont existé entre la révolution industrielle britannique du XVIII ${ }^{e}$ siècle et l'industrie de l'armement demeurent bien plus obscurs. Le complexe militaro-industriel n'émerge réellement que dans la seconde moitié du $\mathrm{XIX}^{\mathrm{e}}$ siècle lorsque la production se concentre. Auparavant les modèles productifs étaient plus diversifiés et artisanaux, même si les armes avaient dès le XVIII ${ }^{\mathrm{e}}$ siècle modelé une société armée et industrialisée. Le livre est construit en trois parties équilibrées qui explorent chacune un des «modes d'existence» des armes à feu. Le livre est riche, très informé sur l'historiographie, même s'il porte essentiellement sur le Royaume-Uni et son Empire ${ }^{2}$. Dans la première partie "The industrial life of guns ", l'auteure suit l'évolution de la fabrication des armes à feu au XVIII ${ }^{\mathrm{e}}$ siècle, elle reconstitue le rôle capital de l'État britannique, mais aussi l'identité des fabricants et les modes d'organisation de la production.

$4 \quad \mathrm{Au} \mathrm{XVIII}{ }^{\mathrm{e}}$ siècle, il est souvent difficile de distinguer les fabricants d'armes des autres ateliers de petite métallurgie situés autour de Birmingham. La thèse de l'auteure est que la fabrication des armes était étroitement associée à d'autres activités diverses, qu'elle a modelé de nouvelles pratiques fondées sur l'interchangeabilité des pièces, et favorisé des liens entre sciences, arts, finances et industrie. La fabrication des armes inaugure en effet des innovations appliquées dans d'autres secteurs, de même que l'effort de guerre ne cesse de soutenir l'ensemble du tissu industriel britannique. En 1815 la Grande-Bretagne est ainsi devenue un vaste dépôt d'armes, une importante fabrique existait près de Londres et de nombreux petits fabricants étaient dispersés dans la région de Birmingham. Bien avant l'ère du grand machinisme et des usines concentrées, ces fabricants ont produit des millions d'armes qui furent échangés à l'échelle du monde en remodelant les rapports de force et de domination à toutes les échelles. Le gouvernement encourage cette production de masse, notamment à la faveur de la guerre de 7 ans, de la guerre d'indépendance américaine, ou des guerres révolutionnaires et napoléoniennes contre la France. Ces conflits ont introduit de nombreux bouleversements dans ce secteur d'activité, une véritable révolution dans la fabrication qui a servi de terreau et de carburant à la «révolution industrielle anglaise ".

5 La seconde partie explore ensuite "The social life of Guns ", c'est-à-dire les usages sociaux de ces armes à feu produites en masse. Les acteurs de l'époque n'utilisaient pas les armes comme nous et ne leur accordaient pas les mêmes significations, tout dépendait du contexte. Ainsi en Afrique de l'Ouest, les armes n'étaient pas destinées qu'à la guerre, elles étaient aussi des symboles de prestige utilisés lors des cérémonies ou pour des offrandes diplomatiques. Le désintérêt pour les nouvelles armes à feu pourtant plus performantes s'explique précisément par ces conditions d'utilisation 
variables et par le sens que les acteurs leur accordaient. Les armes n'étaient pas seulement utilisées pour faire la guerre, elles étaient aussi des moyens d'échange, des biens de valeur aux fonctions multiples. Dans un contexte de pénurie monétaire, les armes pouvaient ainsi servir de substituts et d'instruments de crédit. Mais les armes étaient aussi des auxiliaires de la politique de l'État en faveur de la propriété privée; des instruments de terreur d'un genre nouveau qui accompagnent la criminalisation des classes populaires et certaines de leurs coutumes. Dans cette partie, l'auteure examine les divers types de signification et d'usage des armes à feu dans le monde britannique - en métropole où l'État encadre de plus en plus leur possession - comme dans les diverses parties de l'Empire où elles accompagnent et remodèlent l'expansion coloniale, que ce soit dans les contacts avec les populations d'Amérique du Nord ou d'Asie.

6 La troisième et dernière partie examine enfin la «Moral Life of Guns » en étudiant les débats qui n'ont cessé d'accompagner et de modeler la fabrication et le commerce des armes après 1815. Elle revient sur le désaveu de Galton et les liens entre croyances religieuses et engagements dans l'activité de fabrication des armes. Elle suit la manière dont la fabrication des armes s'est transformée au fil des guerres industrielles modernes, creusant le fossé entre l'Europe et les autres régions du monde. L'auteure offre ainsi une passionnante synthèse de l'évolution du secteur de l'armement jusqu'à nos jours et renouvelle l'analyse de l'avènement du complexe militaro-industriel qui s'impose au $\mathrm{XX}^{\mathrm{e}}$ siècle. Elle étudie aussi l'opposition au commerce des armes et ses ambivalences : à la fin du XXe siècle, la Grande-Bretagne fait voter des lois interdisant la possession des armes à feu tout en restant l'un des principaux producteurs mondiaux.

7 Cette grande fresque de la modernité relue au prisme des armes à feu, depuis leur fabrication jusqu'à leur consommation en passant par leurs diverses modalités de circulation, ouvre de nombreuses perspectives originales sur les origines de l'industrialisation et de l'expansion impériale outre-Manche. Elle contribue aussi à extraire l'histoire des armes du seul champ des spécialistes d'histoire militaire et des passionnés d'armes pour en faire un observatoire qui éclaire, à l'échelle globale, les origines de l'industrialisation du monde, les imaginaires de la violence, comme l'édification de nouveaux rapports de domination. Il s'agit là d'une véritable histoire totale de la modernité pensée au prisme d'une approche au ras du sol. Par ailleurs, et au-delà des seuls enjeux historiographiques, l'auteure revendique une histoire radicale et critique, et n'hésite pas à discuter d'enjeux actuels aussi vifs que le rôle et la place des technologies dans nos vies, les liens intimes entretenus entre l'industrialisme et le militarisme, ou encore le poids variable de la culture des armes dans les diverses régions du monde.

\section{NOTES}

1. Priya Satia, Spies in Arabia: The Great War and the Cultural Foundations of Britain's Covert Empire in the Middle East, New York, Oxford University Press, 2008 ; « Centralité des marges. Les campagnes 
britanniques au Moyen-Orient pendant la Grande Guerre ", Annales. Histoire, Sciences Sociales, vol. $71^{\mathrm{e}}$ année, 2016, p. 87-126.

2. En France les travaux importants de Patrice Bret ont également montré combien les recherches militaires entre 1760 et 1830 ont eu d'importantes retombées industrielles : L'État, l'armée, la science. L'invention de la recherche publique en France (1763-1830), Rennes, Presses universitaires de Rennes, coll. Carnot, 2002. 